\title{
SELECTIVITY OF POLYELECTROLYTE MEMBRANES
}

\author{
O. KEDEM and G. TANNY \\ Laboratory of Membranes \& Bioregulation, The Weizmann Institute of Science, Rehovot, Israel
}

\begin{abstract}
The major factors contributing to the selectivity of charged synthetic membranes are discussed. Transport through porous polyelectrolyte membranes, containing water-filled interstices, can be predicted approximately from Donnan equilibria, representing the interaction between polyelectrolyte and counter-ions by "ion-binding". A simple relation between permselectivity and maximal salt rejection in hyperfiltration is given for porous aqueous membranes. The role of the streaming potential, especially in the filtration of mixed electrolyte, is demonstrated.

Dense polyelectrolyte membranes which swell only slightly in water, give much higher salt rejection than predicted for their permselectivity, according to the Donnan-based relation mentioned: they comprise a separate non-aqueous phase.

Porous membranes, swollen with an organic solvent show very high permselectivity at low charge density. Ion-specificity can be imparted to them by specific complexing agents. It is shown that the change in the dissociation between fixed ionic site and mobile counter-ion by the ligand, may be more important than the change of ion-uptake by the membrane, from the mixed aqueous solution.
\end{abstract}

\section{INTRODUCTION}

The rather odd history of polyelectrolyte membranes is reflected in the variety of terms used for these systems and their properties. The first analysis of "fixed charge" membranes ${ }^{1,2}$ was given in an effort to understand the striking selectivity of biological membranes, especially the electric potential maintained between solutions of different compositions. Though this was a very stimulating and fruitful step for biomembrane research, its main continuation is in membrane technology. The first permselective membranes, including the term "permselectivity", also came from physiologically oriented research. ${ }^{3}$ After the introduction of electrodialysis as a separation process, methods of preparation were developed and the product referred to as "ion-exchange membranes". The alternative membrane process for water purification and desalination which is currently in intense development is reverse osmosis (hyperfiltration).

Thick ion-exchange membranes were amongst the first shown to be capable of high salt rejection, ${ }^{4}$ but the fluxes were very low. Thus these membranes were neglected after the first practical reverse osmosis membranes were fabricated from neutral membrane material, cellulose acetate, with a special asymmetric structure. ${ }^{5}$ Somewhat later, charged membranes of a practical nature were developed. $^{6-9}$ Thus it is now recognized that polyelectrolyte membranes are an important class of reverse osmosis membranes.

The purpose of this lecture is not a systematic review, but a discussion of the main physico-chemical factors contributing to the selectivity of changed synthetic membranes. In this somewhat subjective discussion our own results are used more than they might deserve in a general survey of the field.

\section{THE SYSTEM}

The membrane consists of a network of polymer chains containing covalently bound ionizable groups and a certain volume fraction of water. The "fixed" ionizable groups on the matrix may be introduced into the membrane either as preformed polyelectrolytes or by a suitable reaction, creating functional groups on the polymer network. We may list the phenomena to be dealt with as follows: at the surfaces of contact with the solutions Donnan equilibria are established, giving rise to discontinuity of both ion concentrations and electric potentials. More accurately, very steep gradients exist in the double layer at the surface, however, their detailed profiles are not relevant in the present context. Within the membrane, ion profiles also exist in the aqueous regions about the polyelectrolyte chains, while electrostatic and short range forces may contribute to binding the counterion to the fixed charge site as an ion-pair. If pressure is applied to one of the compartments, water is forced through the membrane and the accompanying drag on the counterions creates a macroscopic electric field parallel to the water flow. For membranes of high charge density and/or low water content, thermodynamic and frictional interactions with the matrix may exist for both counterions and coions.

\section{POROUS CHARGED MEMBRANES}

For an estimate of the relative importance of these phenomena, let us start with the simplest systems: rather loose membranes in which the average distance between polyelectrolyte chains, (or the "pore radius"), is of the order of, or smaller than the thickness of the ionic atmosphere $(1 / \kappa)$, but substantially larger than the ionic radii. The influence of polyions in solution on both thermodynamic and kinetic properties of the small ions has been extensively studied, in theory and experiment. The detailed theoretical analyses resulting from this work has not yet been applied to charged membranesconsidering the uncertainties of membrane structure it may indeed not be justified. Returning to the earlier descriptions of polyelectrolyte-ion interaction, one may regard part of the ions as "bound" and all others as "free". This means that the ideal fixed charge model, allowing for convection, is assumed to be valid with an effective charge density smaller than the ion exchange capacity.

It is instructive to write the Donnan equilibrium for the free ions as follows:

$$
\begin{gathered}
\bar{c}_{1}=X+\bar{c}_{2} \quad \text { (electroneutrality) } \\
\left(\bar{c}_{1} / \phi_{w} c_{s}\right)\left(\bar{c}_{2} / \phi_{w} c_{s}\right)=K_{1} x K_{2}=1 ; \\
\bar{c}_{2} / \bar{c}_{1}=K_{2} / K_{1}=K_{2}{ }^{2}
\end{gathered}
$$

where the bar refers to the membrane phase, $\phi_{w}$ is the 
Table 1. Transport numbers and salt rejection for charged collodion membranes

\begin{tabular}{|c|c|c|c|c|c|c|}
\hline $\begin{array}{c}\text { Membrane } \\
\text { No. }\end{array}$ & $\begin{array}{l}\text { Sign of fixed } \\
\text { charges }\end{array}$ & $\begin{array}{c}\text { Salt } \\
\text { solution }\end{array}$ & $\begin{array}{c}\text { Concentration } \\
(N)\end{array}$ & $\begin{array}{l}\text { Coion transport } \\
\text { number, } \tau_{2}\end{array}$ & $\begin{array}{l}1-R_{\infty} \\
\text { (eqn 5) }\end{array}$ & $\begin{array}{c}1-R_{\infty} \\
\text { (experimental) }\end{array}$ \\
\hline \multirow[t]{2}{*}{1} & Positive & $\mathrm{KCl}$ & $10^{-2}$ & 0.04 & 0.39 & 0.40 \\
\hline & & & $3 \times 10^{-2}$ & 0.47 & 0.98 & 0.72 \\
\hline \multirow[t]{3}{*}{2} & Positive & $\mathrm{KCl}$ & $10^{-2}$ & 0.06 & 0.47 & 0.29 \\
\hline & & & $3 \times 10^{-2}$ & 0.08 & 0.54 & 0.47 \\
\hline & & & $5 \times 10^{-2}$ & 0.13 & 0.65 & 0.62 \\
\hline \multirow[t]{2}{*}{3} & Negative & $\mathrm{KCl}$ & $5 \times 10^{-3}$ & 0.03 & 0.34 & 0.27 \\
\hline & & & $5 \times 10^{-2}$ & 0.30 & 0.91 & 0.84 \\
\hline 4 & Negative & $\mathrm{Et}_{4} \mathrm{NCl}$ & $5 \times 10^{-3}$ & 0.05 & 0.47 & 0.40 \\
\hline
\end{tabular}

volume fraction of water, $X$ the effective charge density and subscripts 1 and 2 refer to counterion and coion respectively. The strongest effect of the fixed charges is the reduction of $K_{2} / K_{1}$.

As mentioned above, charged membranes are used for the removal of salt from aqueous solution in two modes of operation: reverse osmosis and electrodialysis. The ability of membranes to allow the passage of water while retaining the salt is traditionally referred to us as semipermeability while the preferential passage of electric current by ions of one sign only is called permselectivity (cf. definitions are summarized in Fig. 1). From a qualitative consideration of the processes and the Donnan equilibria one can show that with a given charge density it is easier to achieve near-ideal permselectivity than semipermeability.
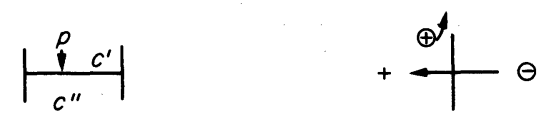

$$
\begin{array}{ll}
\text { Semipermeable } & \text { Permselective } \\
\text { Reflection coefficient, } \sigma & \text { Transport number, } \tau_{ \pm} \\
R O: R_{\infty}=1-\frac{c^{\prime \prime}}{c^{\prime}}=\sigma & \text { ED: } F J_{ \pm} / I=\tau_{ \pm} \\
\text {Ideal }: \quad \sigma \rightarrow 1 & \text { Ideal }: \tau_{ \pm} \rightarrow 1
\end{array}
$$

Fig. 1. Semipermeability vs permselectivity.

The quantitative analysis of flows in hyperfiltration is greatly simplified by the fact that at high flow rate the concentration profile within the membrane becomes flat near the "feed" (high-pressure) boundary. ${ }^{10}$ Ion flows are then completely determined by the concentration (fixed by the Donnan equilibrium at the surface), convection and the streaming potential dictated by electroneutrality. For the salt rejection at high flow rates, $R_{\infty}$, one obtains: ${ }^{11}$

$$
\begin{gathered}
1-R_{\infty}=1-\sigma=K_{2} /\left(t_{1}+t_{2} K_{2}^{2}\right) \\
t_{1}=\frac{u_{1}}{u_{1}+u_{2}} \quad t_{2}=\frac{u_{2}}{u_{1}+u_{2}}
\end{gathered}
$$

where $u_{1,2}$ are the mobilities of the ions in free solution and hence $t_{1,2}$ their transport numbers. The ratios between the ion mobilities in the membrane were assumed to be equal to that in free solution. $\dagger$ The transport number of the coion in the membrane, $\tau_{2}$, is

$$
\tau_{2}=\frac{\bar{u}_{2} \bar{c}_{2}}{\bar{u}_{1} \bar{c}_{1}+\bar{u}_{2} \bar{c}_{2}}=\frac{t_{2} \bar{c}_{2} / \bar{c}_{1}}{t_{1}+t_{2} \bar{c}_{2} / \bar{c}_{1}} .
$$

$\uparrow$ This assumption may be unjustified for certain electrolytes. A full discussion of this point will be given elsewhere. ${ }^{12}$
Hence

$$
\frac{\tau_{2}}{t_{2}} / \frac{1-\tau_{2}}{1-t_{2}}=\bar{c}_{2} / \bar{c}_{1}=K_{2}^{2} .
$$

Equation (4) shows that for permselectivity the deviation from ideality diminishes with $K_{2}^{2}$. Elimination of $K_{2}$ from eqns (2) and (4) gives the correlation between the key parameters of the two separation processes:

$$
1-\sigma=\left[\frac{\tau_{2}\left(1-\tau_{2}\right)}{t_{2}\left(1-t_{2}\right)}\right]^{2} .
$$

For a solution of, e.g. $\mathrm{NaCl}$, this means that a porous cation-exchange membrane which reduces the transport number of $\mathrm{Cl}$ to $2 \%$ is expected to give only $71 \%$ maximal salt rejection in reverse osmosis.

Table 1 shows that for porous collodion membranes in which a polybase was adsorbed and crosslinked, eqn (5), though based on a very simple model, is a reasonable approximation. This does not necessarily mean that the distribution of the free ions in the interstices of the polyelectrolyte matrix is indeed homogeneous. A more detailed calculation of salt rejection in charged channels showed that the result may be surprisingly insensitive to deviations from homogeneity. ${ }^{14}$ In this study accurate numerical computations were carried out on a model of very simple geometry: a series of plane-parallel aqueous channels cutting through an ion-exchange gel. Since all flows are parallel to the walls of the channels, the equilibrium ion distribution ${ }^{15}$ may be assumed to be undisturbed. Superimposing ion-convection by pressureinduced viscous flow, and the consequent streaming potential, one obtains the velocity profile of the ions. The average flow in a plane parallel to the membrane is given by the average product of ion concentration and velocity: $J_{i}=\left\langle v_{i} c_{i}\right\rangle$. With the assumption of homogeneity we would calculate in effect the flow from the product of the averages, $\left\langle v_{i}\right\rangle\left\langle c_{i}\right\rangle$. Figure 2 shows that the flux density may vary as a function of distance from the wall, reflecting variations in both concentrations and velocities. ${ }^{15}$ In fact in the case shown, the counterions near the wall are moving in the direction opposite to that of the filtration flow, pushed back by the streaming potential. Nevertheless, in the example chosen, the ratio between the "homogeneous" and the correct average was only from a few per cent to $\sim 10 \%$ different from unity. This is of course only an illustration of the problem and not a general proof.

\section{THE STREAMING POTENTIAL IN HYPERFLLTRATION}

The electric potential built up during hyperfiltration of a single electrolyte accelerates coions and retards counterions, maintaining electroneutrality. At high flow rates the 


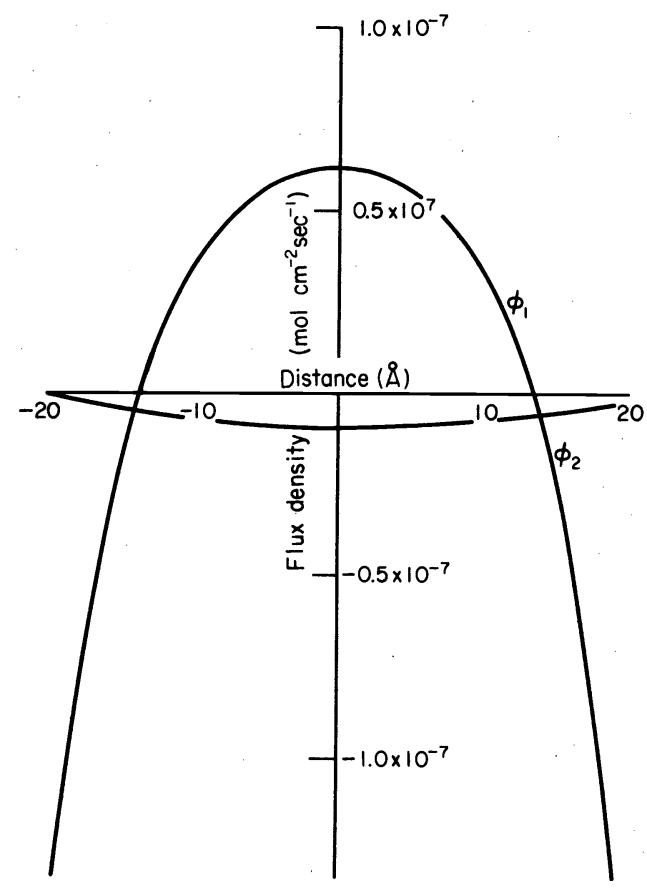

Fig. 2. Profiles for the counterion, $\phi_{1}$ and coion, $\phi_{2}$, flux densities in a charged 40 Å pore (Fig. 5 of Ref. 14).

potential is a linear function of volume flow and its determination is a convenient way for further characterization of the membrane. Figure 3 shows the streaming potential measured with a pair of reversible electrodes across the hyperfiltration membrane (porous collodion + polybase). ${ }^{16}$

The acceleration of the coions is expressed in eqn (2) by the denominator $t_{1}+t_{2} K_{2}{ }^{2}$, which is smaller than one. In the absence of a field, the salt flow $J_{s}$, identical to the coion flow, would be simply $J_{s}=\bar{c}_{2} J_{v}$, with $\bar{c}_{2}$ the coion concentration near the feed boundary and $J_{v}$ the volume flow. Since the concentration of the "product" in hyperfiltration, $c^{\prime \prime}$, must be equal to $J_{s} / J_{v}$, the rejection without a field would be:

$$
R_{(E=0)}=1-\left(\frac{c^{\prime \prime}}{c^{\prime}}\right)_{E=0}=1-\left(\frac{\bar{c}_{2}}{c^{\prime}}\right)=1-K_{2} .
$$

In fact, salt rejection is partly spoiled $\left(1-R>K_{2}\right)$ by the field created by counterion convection.

One might construct a membrane containing shunt pathways for the counterions: patches of high counterion conductance and low water-permeability. Such a "permeability mosaic" would increase the salt rejection, but its practical attractivity is limited by the fact that the upper limit for porous membranes, $1-K_{2}$, is not close enough to unity. ${ }^{17}$

The role of the streaming potential is particularly apparent in the filtration of mixed electrolyte solutions. ${ }^{18}$ An extreme example is shown in Table 2. Both $\mathrm{Cu}^{2+}$ and $\mathrm{H}^{+}$are coions in the positive membranes used. Comparing the mixture to each of the solutes in separate solutions, at the same concentrations, one sees that the rejection of $\mathrm{Cu}^{2+}$ is increased by the presence of $\mathrm{H}^{+}$and at the same time $R_{\mathrm{H}^{+}}$is decreased and becomes negative. A more surprising effect was found in mixtures of counterions of different valency and mobility, and explained by Dresner ${ }^{19}$ by the influence of the streaming potential: the divalent counterion which is of course taken up preferentially by the charged membrane, may be completely rejected in the presence of a second fast univalent counterion. This mechanism is illustrated in Fig. 4,

Table 2. Hyperfiltration of copper chloride-hydrochloric acid mixtures in a positively charged collodion membranes (data taken from Ref. 18)

\begin{tabular}{lrrl}
\hline \multicolumn{2}{c}{ Feed concentration } & \multicolumn{2}{c}{ Rejection } \\
\hline $\mathrm{HCl}$ & $\mathrm{CuCl}_{2}$ & $\mathrm{R}_{\mathrm{H}} \%$ & $\mathrm{R}_{\mathrm{Cu}} \%$ \\
\hline 0.05 & & 6.5 & \\
& 0.05 & & 28 \\
0.05 & 0.05 & -14.1 & 43.2 \\
0.01 & 0.05 & -46.2 & 37.9 \\
0.05 & 0.01 & 1.6 & 63.4 \\
0.1 & & 1.5 & \\
0.1 & 0.1 & & 17 \\
& 0.1 & -6.7 & 21.3 \\
\hline
\end{tabular}

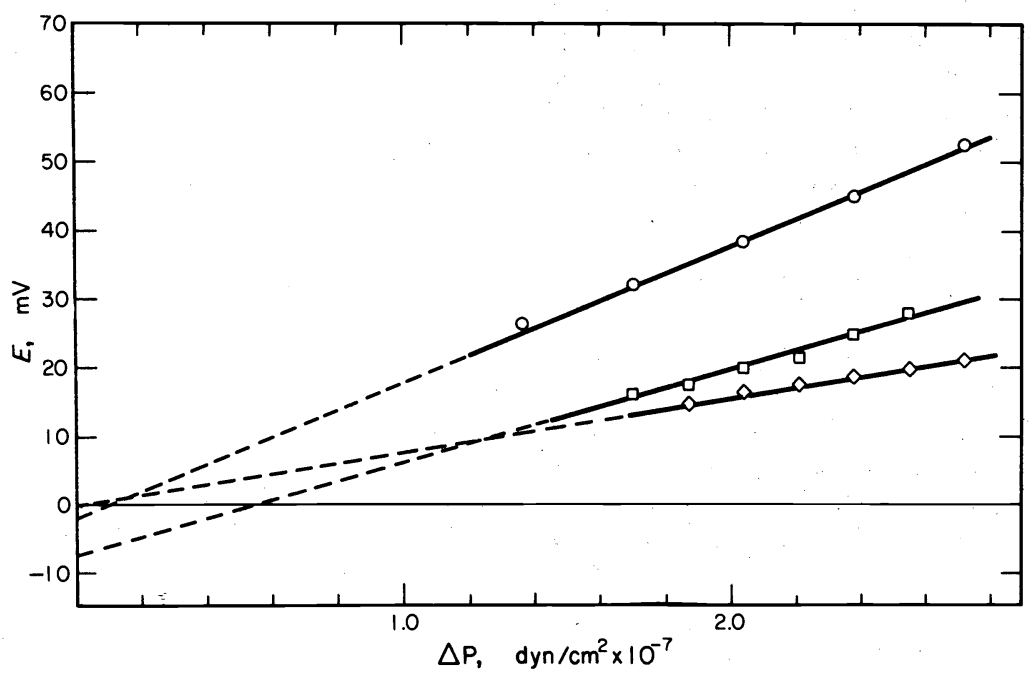

Fig. 3. Hyperfiltration streaming potential as a function of pressure for a positively charged collodion-PVA membranes (Fig. 2 of Ref. 16). (O) $-10^{-2} \mathrm{~N} \mathrm{KCl} ;(\square)-3 \times 10^{-2} \mathrm{~N} \mathrm{KCl} ;(\diamond)-5 \times 10^{-2} \mathrm{~N} \mathrm{KCl}$. 
$\bar{c}_{2}$

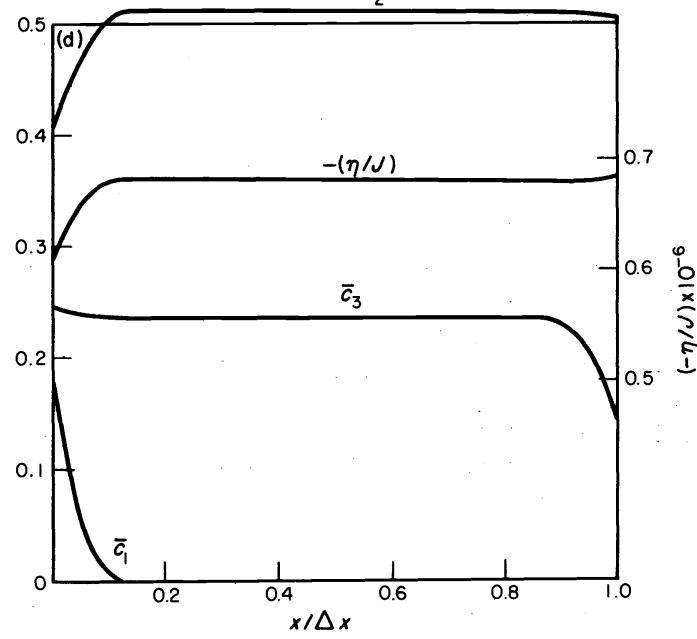

Fig. 4. Concentration and electric field profiles in a cation-exchange membrane during hyperfiltration of an equinormal $\mathrm{HCl}-\mathrm{MgCl}_{2}$ mixture. $C_{1}^{\prime} / C_{3}^{\prime}=0.5,\left|C_{3}^{\prime}\right| X \mid=0.1$.

calculated for a negative membrane: ${ }^{20}$ the electric field is higher the lower the concentration of $\mathrm{Mg}^{2+}$. Starting from the feed boundary, the field increases and the concentration of $\mathbf{M g}^{2+}$ decreases. Hence the magnesium ion penetrates the membrane only to a certain depth and is prevented from crossing to the product compartment.

In summary, in porous charged membranes, Donnan exclusion, convection and streaming potential are the major factors determining selectivity.

\section{DENSE AND NON-AQUEOUS CHARGED MEMBRANES; ION SPECIFIC MEMBRANES}

Table 3 shows an example from a different family of membranes, consisting of water-insoluble polyelectrolytes which can be cast into dense films, swelling only slightly in water. The relation between transport number and salt rejection was chosen as a test for the general behaviour of the membrane. Column 4 gives $1-R_{\infty}$ predicted from the transport numbers according to eqn (5). It is clear that Donnan exclusion alone is not the basis for selectivity, and it is essential to allow for short-range interactions. Commercial ion-exchange membranes whose water permeability is purposely kept low by a high degree of cross-linking or by mixing with uncharged polymers, seem to belong to the same general class. Their high salt-rejection has already been mentioned. ${ }^{4}$

In the absence of any specificity two major effects have to be considered. Firstly, the membrane phase as a whole constitutes a non-aqueous medium and therefore the standard state of electrolytes is not equal to that in aqueous solution. In other words, the solubility of salts will be decreased apart from the Donnan exclusion; secondly, during migration of ions in the membrane, friction between ions and matrix is not negligible as compared to the ion/water friction. This means that the ions are not swept along with the water to the same degree as in convective capillary flow. Both factors will increase salt rejection ${ }^{21}$ while the influence on the transport numbers is not predictable without more detailed information on the state of the ions. A formal framework for the characterization of the kinctic interactions between all species in terms of mutual frictions was suggested by Spiegler. ${ }^{22}$ Meares and coworkers ${ }^{23}$ and a number of other groups have used this approach for an extensive experimental and theoretical analysis of membrane behaviour, which has been reviewed ${ }^{23,24}$ previously.

In the following we shall address ourselves to the question of how short range interactions can be used in order to create selectivity.

In the separations considered so far the membrane had to distinguish between ions and water, cations and anions, or mono- and multivalent ions of the same sign. The selectivity which is most difficult to achieve is that distinguishing between ions of the same charge-a classic example being $\mathrm{K}^{+} / \mathrm{Na}^{+}$. This has long been a challenge to membrane development both because of its practical importance and because biological membranes show near-ideal specificity.

"Ion-specific" membranes may be applied in separation processes or in measuring electrodes, and the requirements in the two cases are not identical. A specific electrode "responds correctly" to the activity of the examined ion in solution, if the electric potential established between a reference and the test solution is linearly dependent on, or at least uniquely determined by, the chemical potential of the test-ion in the solution. This should hold even in the presence of other electrolytes. The membrane potential, $\Delta \psi$, is given by

$$
\Delta \psi=-\sum_{i} \frac{\tau_{i}}{F Z_{i}} \Delta \psi_{i}^{c}
$$

where $Z_{i}$ is the charge of ion $i$ and $\Delta \psi_{i}^{c}$ the difference of its electrical potential between the two solutions. For a satisfactory electrode the membrane has to be permeable to one ion much more than to all others. The total conductivity may be quite small-only large enough in order not to make the electrode too sensitive to stray potentials. On the other hand, in a separation process a transport number significantly smaller than one may still be useful, but the absolute values of ionic conductances have to be high.

A plausible mechanism for ion-specificity of a charged membrane would seem to be specific binding of counterions to the charged sites. Two serious difficulties prevent

Table 3. Transport numbers and salt rejection of $\mathrm{NaCl}$ solutions in sulphonated polysulphone membranes

\begin{tabular}{crccc}
\hline \multicolumn{1}{c}{$c^{\prime}$} & \multicolumn{1}{c}{$c^{\prime \prime}$} & $\left(\tau_{+}\right)_{\text {app }}$ & $\begin{array}{c}1-R_{\infty} \\
\text { (from eqn (5)) }\end{array}$ & $\begin{array}{c}1-R_{\infty} \text { with feed } c_{1} \\
\text { (experimental) }\end{array}$ \\
\hline $9.34 \times 10^{-3}$ & $9.5 \times 10^{-4}$ & 0.975 & 0.32 & 0.01 \\
$1.86 \times 10^{-2}$ & $1.92 \times 10^{-3}$ & 0.965 & 0.38 & \\
$4.54 \times 10^{-2}$ & $4.68 \times 10^{-3}$ & 0.98 & 0.29 & \\
$9.6 \times 10^{-2}$ & $9.3 \times 10^{-3}$ & 0.915 & 0.57 & 0.02 \\
$1.9 \times 10^{-1}$ & $1.86 \times 10^{-2}$ & 0.884 & 0.66 & \\
$4.70 \times 10^{-1}$ & $4.54 \times 10^{-2}$ & 0.828 & 0.77 & 0.055 \\
\hline
\end{tabular}


the construction of separation-membranes according to this principle. A rate of migration limited by dissociation-association of a specific site can hardly be high enough. Moreover, higher uptake of the desired ion from solution is counteracted, and often overcompensated, by lower mobility in the membrane. The failure of a number of attempts at ion-specific electrodialysis demonstrated this overcompensation. There is however no general law relating the distribution coefficients and rates of "hopping". If overall fast transport is not necessary it may be possible to find a favourable combination of parameters. This is apparently the case in ion-specific glass-electrodes. ${ }^{25}$

Some of the currently used membrane electrodes consist of "immobilized" solvent with dissolved specific complexing agents. The role of the solid support and the fixed charges it carries has not always been stated explicitly.

Using plasticized cellulose acetate with chemically bound charges ("Brilliant Orange" dye) we were able to control the volume fractions of polymer matrix and solvent-plasticizer and the charge density. ${ }^{26} \mathrm{~A}$ remarkable result found with these membranes was the high permselectivity obtained with a small charge density (cf. Table 4). A fraction of a millimole per litre gave ideal permselectivity with solutions up to $0.1 \mathrm{~N}$. At the same time coion exclusion was shown to be far from ideal. The main explanation for this high selectivity is to be found not in the surface equilibria between membrane and external solution, but in the membrane itself. We are dealing with a medium of rather low dielectric constant in which most of the ions are associated to ion-pairs, and therefore ionic conductances and transport numbers are determined by the small fraction of free ions. Since mobilities of small ions are of the same order, the transport number of the coion is essentially determined by the ratio between free coions and free counterions. As a consequence of electroneutrality

$$
\frac{c_{2}^{*}}{c_{1}^{*}}=\frac{c_{2} / X^{*}}{1+c_{2}^{*} / X^{*}}
$$

where the asterisks denote free ions and fixed charges. Setting activities equal to concentrations at this high dilution and assuming small dissociation

$$
c_{2}^{*} / c_{1}^{*}<c_{2}^{*} / X^{*}=\left(K^{s} / K^{f}\right) \bar{c}_{2} / X
$$

where $K^{s}$ and $K^{f}$ are the dissociation constants for coion/counterion and for fixed site/counterion respectively. $\bar{c}_{2}$ and $X$ refer to the total concentrations and fixed charge density. Equation (7) shows that the permselectivity of such a membrane depends on the relative dissociation of two types of ion pairs. It is thus influenced by the nature of these groups and ions ${ }^{26,27}$ and of the plasticizer-solvent. In these circumstances, permselectivity cannot be predicted from charge density and salt uptake alone.

These slightly charged and highly permselective cation exchange membranes become ion-specific for $\mathrm{K}^{+}$upon addition of valinomycin, a highly specific K-complexing agent. Once again, the heart of the phenomenon is not a change in the surface equilibria, but a shift in dissociation within the organic phase. In the presence of potassium and an excess of sodium in the aqueous solution, both ions enter the membrane. While there is a substantial preference for potassium without valinomycin, sodium is not excluded. A small quantity of valinomycin, much less than equivalent to the fixed charges in the membrane changes the ratio of $\mathrm{K}^{+}$to $\mathrm{Na}^{+}$in the membrane very lightly, but gives rise to practically ideal $\mathrm{K}^{+}$-response of the electrode. These membrane compositions and potentials show that the average mobility, or fraction of free ions, was increased for $\mathrm{K}^{+}$as compared to $\mathrm{Na}^{+}$. Valinomycin is an uncharged depsipeptide and one must conclude that the $\mathrm{K}^{+}(\mathrm{Val})$ complex is less associated to the fixed charges than $\mathrm{K}^{+}$or $\mathrm{Na}^{+}$. This is in fact to be expected following the analysis of Simon and Morf.$^{28}$ The spherical shell formed by the ligand increases markedly the radius of closest approach, decreasing the electrostatic energy gain by ion-pair formation, and the free complex-ion is very well soluble in the solvent-plasticizer.

The opposing effects of binding on uptake and average mobility are here avoided: the highly specific binding between $\mathrm{K}^{+}$and valinomycin has indeed a rather long relaxation time, but the process of ion migration does not require dissociation-association of this complex. The binding between fixed site and counterion is not enhanced but hindered by the complexation.

The study of polyelectrolyte membranes with waterfilled interstices draws upon a large and detailed store of knowledge on polyelectrolyte solutions. Though membrane-theory so far has not developed to the refinement of existing theory for transport in solutions, the general principles are applicable. A similar experimen-

Table 4. Permselectivity and $\mathrm{K}^{+}$-specificity of slightly charged cellulosic membranes containing organic solvent

\begin{tabular}{lcccc}
\hline Membrane & $\begin{array}{c}\text { Charge density } \\
(\mathrm{mmol} / \mathrm{l})\end{array}$ & $\begin{array}{c}\text { Potentiometric } \neq \\
\text { slope } \\
(\mathrm{mr} / \mathrm{dec}\end{array}$ & $\begin{array}{c}\text { Valinomycin }) \\
\text { concentration } \\
(\mathrm{mmol} / \mathrm{l})\end{array}$ & $\begin{array}{c}\text { Sodium } \\
\text { error } \\
(\%)\end{array}$ \\
\hline Cellulose acetate & & & & \\
(C.A.) & Very small & $25-30$ & 0 & \\
C.A.-CABO $\dagger$ & 0.3 & 59.0 & 0 & \\
C.A.-CABO & 0.6 & 58.5 & 0 & \\
Millipore & 4 & 59.0 & 0 & 8 \\
Millipore & 4 & 59.5 & 0.3 & 1 \\
Millipore & 4 & 59.5 & 0.7 & \\
\hline
\end{tabular}

†C.A. mixed with charged C.A.

$\ddagger$ The potentials across the membrane were determined with calomel electrodes and $\mathrm{KCl}$ solutions, one of which was fixed at $1 \mathrm{mmol} / \mathrm{l}$ while the other varied between $0.1 \mathrm{mmol}-$ $0.1 \mathrm{mmol} / \mathrm{l}$. "Sodium error" was determined in the presence of a 30-fold excess of $\mathrm{NaCl}$.

$\S$ Very small charge density which may originally exist in pure C.A. 
tal and theoretical background on the behaviour of non-aqueous polyelectrolyte solutions would put the understanding of nonaqueous charged membranes on a much firmer basis.

Another important direction for future research is related to the distribution of charges, of hydrophilic and hydrophobic regions within the membrane. In the study of neutral reverse osmosis membranes, the role of the exact mode of phase separation during membrane preparation is increasingly recognized. A similar development for charged nonaqueous membranes would probably be very fruitful.

\section{REFERENCES}

${ }^{1}$ T. Teorell, Proc. Soc. Exp. Biol. Med. 33, 282 (1935); Proc. Nat. Acad. Sci. 21, 152 (1935).

${ }^{2}$ K. H. Meyer and G. F. Sievers, Helv. Chim. Acta, 19, 649, 665, 987 (1936).

${ }^{3} \mathrm{~K}$. Sollner, Electrochemistry in Biology and Medicine, (Edited by T. Shedlovsky), p. 41, Wiley, New York (1955).

${ }^{4} J$. G. McKelvey, K. S. Spiegler and M. R. J. Wyllie, Chem. Eng. Progr. Symp. Ser. 55, 199 (1959).

${ }^{5} S$. Loeb and S. Sourirajan, Advan. Chem. Ser. 38, 117 (1963). ${ }^{6} \mathrm{~J}$. S .Johnson, Jr., in Reverse Osmosis Membrane Research, (Edited by H. K. Lonsdale and H. E. Podall), Plenum Press, New York (1972) and references therein.

'S. G. Kimura, I \& EC Prod. Res. \& Dev. 10, 334 (1971).

${ }^{8} \mathrm{G}$. Tanny and J. Jagur-Grodjinski, Desalination, 13, 53 (1973).
${ }^{9}$ R. Chapurlat, Proc. IV Int. Sym. on Fresh Water From The Sea, Heidelberg, Vol. 4 (1973).

${ }^{10}$ L. Dresner, J. Phys. Chem. 76, 2256 (1972).

${ }^{11}$ E. Hoffer and O. Kedem, Desalination, 2, 25 (1967).

${ }^{12} \mathrm{G}$. Tanny, G. Garza and O. Kedem, in preparation.

${ }^{13}$ O. Kedem, Israel J. Chem. 11, 313 (1973).

${ }^{14}$ R. Simons and O. Kedem, Desalination, 13, 1 (1973).

${ }^{15}$ E. P. George and R. Simons, Aust. J. Biol. Sci. 19, 459 (1966).

${ }^{16} \mathrm{G}$. Tanny and O. Kedem, J. Coll. Int. Sci. 51, 177 (1975).

${ }^{17} \mathrm{G}$. Tanny and E. Hoffer, Permeability mosaic membranes, Sect. ID, O.S.W. Report of O. Kedem \& D. Vofsi, contract No. 14-010001-961, Aug. 71-72.

${ }^{18}$ E. Hoffer and O. Kedem, I \& EC Proc. Des. \& Dev. 11, 226 (1972).

${ }^{19}$ L. Dresner, Desalination, 10, 27 (1972).

${ }^{20} \mathrm{G}$. Garza, Ph.D. Thesis, Weizmann Institute of Science, Rehovot.

${ }^{21}$ K. S. Spiegler and O. Kedem, Desalination 1, 311 (1966).

${ }^{22}$ K. S. Spiegler, Trans. Far. Soc. 54, 1408 (1958).

${ }^{23} \mathrm{P}$. Meares, in Membranes. (Edited by G. Eisenmann), Vol. I, Chap. 2, Marcel Dekker, New York (1972).

${ }^{24} \mathrm{~N}$. Lakashmanarayaniah, Transport Phenomena in Membranes. Academic Press, New York (1969).

${ }^{25} \mathrm{G}$. Eisenmann, in The Glass Electrode. (Edited by G. Eisenmann, R. Bates, G. Matlock and S. M. Friedman), WileyInterscience, New York.

${ }^{26}$ O. Kedem, M. Perry and R. Bloch, Nato Advanced Study Institute on Charged \& Reactive Polymers, France (1974).

${ }^{27}$ J. H. Boles and R. P. Buck, Anal. Chem. 45, 2057 (1973).

${ }^{28}$ W. Simon and W. E. Morf, in Membranes. (Edited by G. Eisenmann), Vol. II, p. 329, Marcel Dekker, New York (1973). 